\title{
Utilisations des plantes désignées comme adventices par les populations riveraines de la forêt classée de Sanaimbo (centre-est de la Côte d'Ivoire).
}

\author{
Touré Awa ${ }^{\text {* }}$, Amani Yao Célestin², Adou Lydie Marie Dominique ${ }^{1}$ \& Ipou Ipou Joseph ${ }^{1}$ \\ 1 Laboratoire de Botanique, UFR Biosciences, Université Félix Houphouët-Boigny, 22 BP 582 Abidjan 22 (Côte d'Ivoire). \\ 2 Institut des Sciences Anthropologiques de Développement, UFR Sciences de l'Homme et de la Société, Université Félix \\ Houphouët-Boigny, 22 BP 582 Abidjan 22 (Côte d'Ivoire) \\ *Auteur pour toute correspondance : awatour93@gmail.com/ Téléphone : +225 07029080/ +22504821669
}

Original submitted in on $20^{\text {th }}$ April 2018. Published online at www.m.elewa.org on $31^{\text {st }}$ July 2018 https://dx.doi.org/10.4314/jab.v127i1.7

\section{RESUME}

Objectifs : En agronomie, les adventices sont considérées comme nuisibles et l'on cherche toujours des méthodes de lutte pour réduire leur impact sur la production des cultures agricoles. Mais comme toute plante, elles peuvent posséder des vertus. Ce travail vise à déterminer les utilisations faites des plantes " adventices » par les populations paysannes des villages environnants la forêt classée de Sanaimbo située au centre-est de la Côte d'Ivoire.

Méthodologie et résultats: La méthode d'approche est une enquête ethnobotanique réalisée auprès des populations. Elle s'est faite par une interview directe sur la base d'un questionnaire préalablement conçu. L'étude a montré que les populations riveraines de la forêt classée de Sanaimbo, connaissent et maintiennent dans leurs champs environ 77 espèces d'adventices qu'elles utilisent à plusieurs fins. Ainsi, elles sont employées à $79 \%$ dans la médecine traditionnelle, $11 \%$ dans l'alimentation et $10 \%$ dans les autres domaines tels que la chasse, l'habitat, la vannerie.

Conclusion et applications des résultats : Le fort taux d'utilisation des mauvaises herbes dans le domaine thérapeutique montre bien, que les populations de ces localités dépendent plus de la médecine traditionnelle et de la phytothérapie que de la médecine moderne qu'elles jugent coûteuse.

Mots clés : Mauvaise herbe, ethnobotanique, forêt classée, centre-est, Côte d'Ivoire

Uses of plants designated as weeds by the riparian populations of the Sanaimbo classified forest (Eastcentral Côte d'Ivoire).

ABSTRACT

Objectives: In agronomy, weeds are considered as pests and methods to reduce their impact on the production of agricultural crops are being researched. But like any plant, weed can also have virtues. This work aims to determine the uses made of "weed" plants by peasant populations in the villages surrounding the Sanaimbo classified forest located in central-eastern Côte d'Ivoire.

Methodology and results: The approach method is an ethnobotanical survey carried out among the populations. It was done by a direct interview based on a previously designed questionnaire. The study showed that populations living near the Sanaimbo Forest Reserve know and maintain in their fields about 77 species of 
weeds that they use for many purposes. Thus, they are used $79 \%$ in traditional medicine, $11 \%$ in food and $10 \%$ in other areas such as hunting, habitat, basketry.

Conclusion and applications of the results: The high rate of weed plants use in the therapeutic area shows that the populations of these localities depend more on traditional medicine and phytotherapy than on modern medicine, which they consider expensive.

Keywords : Weed, Ethnobotany, Classified Forest, Central East, Ivory Coast

\section{INTRODUCTION}

Dans le contexte agronomique, adventice désigne toute plante herbacée ou ligneuse indésirable à l'endroit où elle se trouve, désignée dans le langage courant par l'expression « mauvaise herbe ». Cette notion "mauvaise herbe » est parfois rejetée à cause de la connotation négative pour la plante ainsi désignée (Ipou Ipou, 2005). Cependant, elle est parfaitement adaptée si l'on ne l'applique pas à l'espèce, mais seulement aux individus qui sont indésirables là où ils se trouvent (AFNOR, 1977). Cette notion de "mauvaise " n'a donc pas une valeur absolue. Dans les agroécosystèmes traditionnels au Mexique, Altieri (1987) montre que les paysans maintiennent en association avec la culture, certaines espèces qu'ils appellent « buen

\section{MATÉRIEL ET MÉTHODES}

Situation et caractéristiques du milieu d'étude : La forêt classée de Sanaimbo est à cheval sur les départements de Bongouanou et de Dimbokro (figure 1). Elle se localise entre $6^{\circ} 30^{\prime}$ et $6^{\circ} 40^{\prime}$ de latitude nord et $4^{\circ} 25^{\prime}$ et $4^{\circ} 35^{\prime}$ de longitude ouest. N'gohinou, Soungassi, Adouakouakro et Assahara sont des villages qui se situent autour de cette forêt. Sur le plan administratif, N'gohinou, Adouakouakro et Assahara font partie du département de Bongouanou et Soungassi appartient au département de Dimbokro. Le relief de ces départements est monotone et constitué d'un plateau légèrement incliné monte » (bonnes plantes) et n'éliminent que les « mal monte » (mauvaises plantes). En effet, dans de nombreux systèmes traditionnels en région tropicale, différentes "mauvaises herbes " sont maintenues dans les champs et utilisées à divers fins : nourriture, médecine, cérémonies religieuses, amélioration du sol (Ruthenberg, 1976 ; Gliessman, 1988). Ainsi, dans les localités de N'gohinou, de Soungassi, d'Adouakouakro et d'Assahara, les populations épargnent certaines «mauvaises herbes » dans leurs parcelles cultivées lors des opérations d'entretien. Cette étude a été menée afin de connaître les utilisations faites de ces plantes par ces populations.

de direction Nord-Sud, avec une altitude moyenne supérieure à $150 \mathrm{~m}$. La végétation est constituée de lambeaux de forêt mésophile, et de larges mailles de savanes séparées par des forêts galeries. Il existe à côté de ces végétations naturelles, des végétations liées à l'action anthropique (mosaïques forêt-cultures, mosaïques savanes-cultures, cultures). Le climat est de type tropical humide avec une alternance de 4 saisons dont deux (2) saisons pluvieuses et deux (2) saisons sèches. La hauteur pluviométrique annuelle est de 1155 mm (Kouassi et al., 2013). 


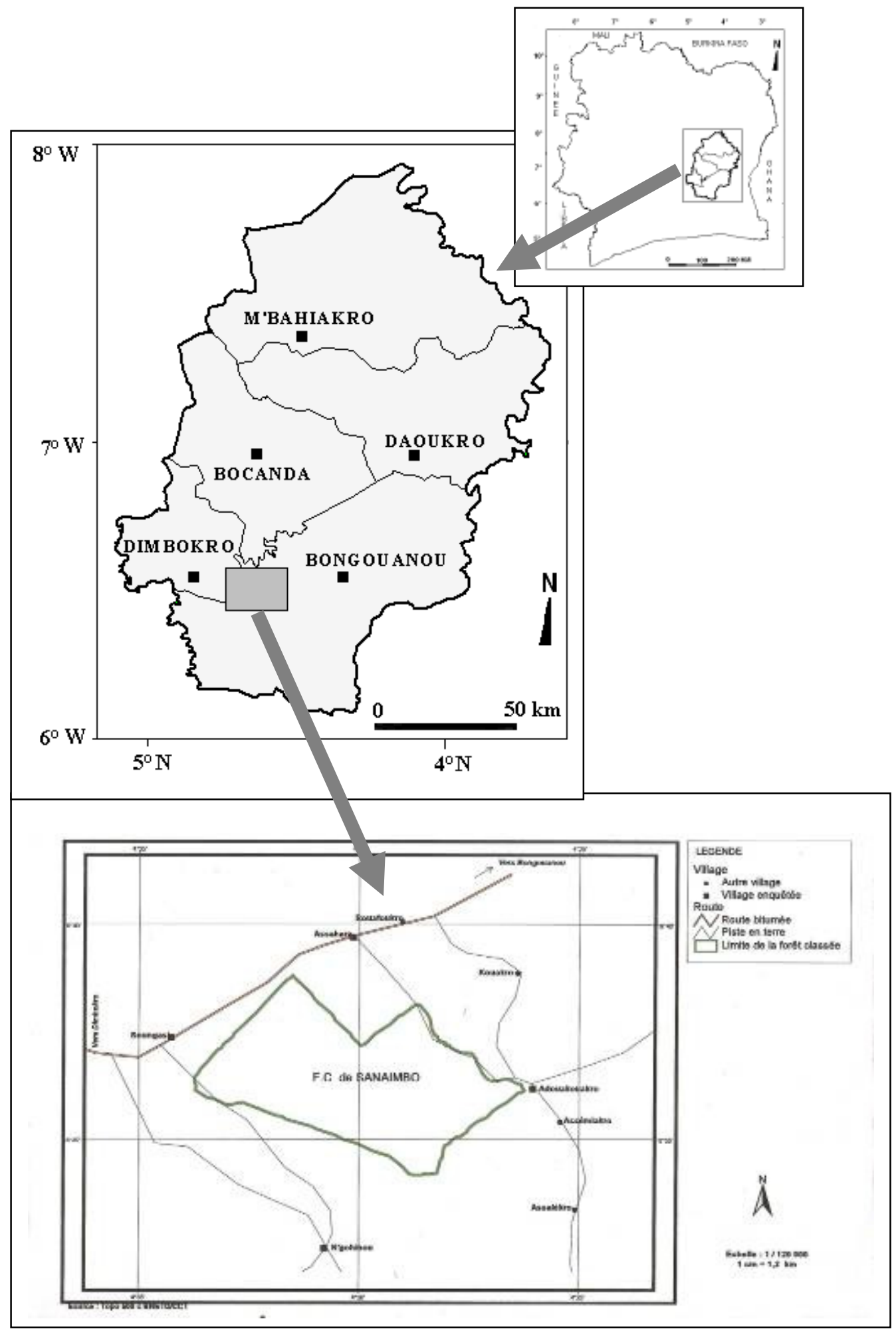

Figure 1 : Localisation des villages enquêtés dans les départements de Bongouanou et Dimbokro (Côte d'Ivoire)

Matériel: Une pré-enquête a été réalisée afin de recenser les adventices fréquemment utilisées par les habitants de ces différentes localités. Ce sont 77 espèces qui ont été recensées et ont fait l'objet d'enquête auprès 
des populations de ces 4 villages (N'gohinou, Adouakouakro, Assahara et Soungassi). Ainsi, le matériel est constitué de ces 77 espèces et des fiches d'enquête (figure 2) conçues pour noter les informations.

Méthode : La méthode d'approche est une enquête réalisée auprès des populations des quatre localités visitées, c'est-à-dire N'gohinou, Soungassi, Assahara et Adouakouakro. Le mode de collecte des informations est une entrevue personnelle basée sur un questionnaire préalablement conçu. Le questionnaire comporte des questions ouvertes et fermées (figure 2). Des personnes appartenant aux deux genres et aux différentes classes d'âge ont été interrogées. L'objectif visé est de recenser et d'analyser les différents domaines d'utilisation actuelle de ces 77 adventices par les populations de ces localités. Les données recueillies sont analysées grâce au logiciel EXCEL 2010. Le traitement des données s'articule autour de deux points. Le premier point consiste à cataloguer les adventices selon leurs différents usages et à les classer selon leurs domaines d'utilisations qui sont l'alimentation, la médecine traditionnelle et d'autres usages (construction, chasse, artisanat, esthétique). Le second point porte sur le calcul des taux d'utilisation des organes végétaux de ces plantes dans les différents domaines d'utilisation.

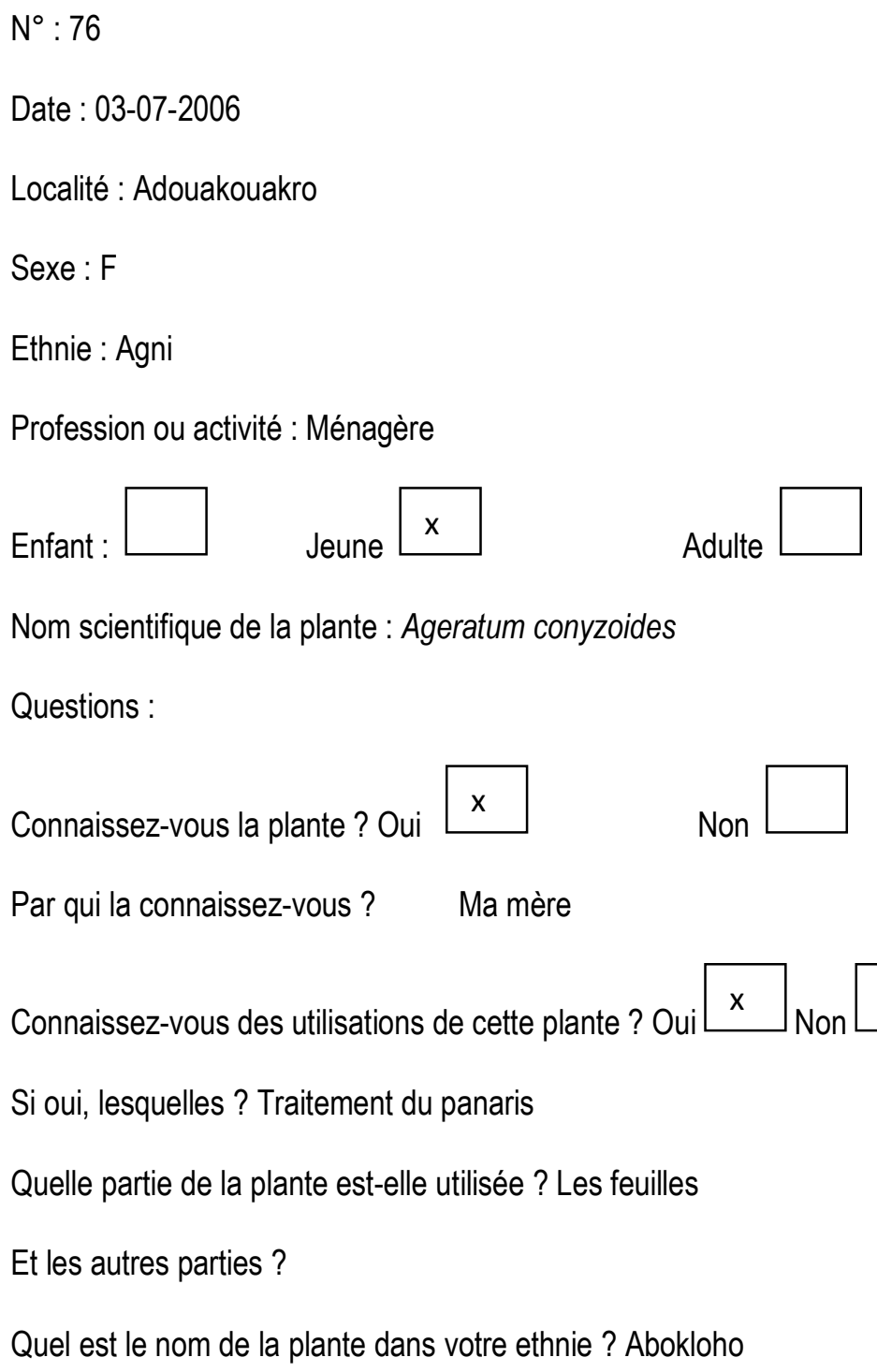

Nom scientifique de la plante : Ageratum conyzoides

Questions :

Connaissez-vous la plante ? Oui

$\mathrm{X}$

Par qui la connaissez-vous ? Ma mère

Non

Si oui, lesquelles ? Traitement du panaris

Quelle partie de la plante est-elle utilisée ? Les feuilles

Et les autres parties?

Quel est le nom de la plante dans votre ethnie ? Abokloho

Figure 2 : Fiche d'enquête ethnobotanique 


\section{RÉSULTATS}

Caractéristiques botaniques des adventices utilisées : Les 77 espèces inventoriées sont reparties en 70 genres appartenant à 39 familles. La figure 3 illustre quelques adventices utilisés par les populations de la zone d'étude. Les familles les plus représentées en nombre d'espèces sont les Euphorbiaceae avec 7 espèces, les Amaranthaceae et les Sapindaceae avec 6 espèces chacune. Dans cette flore, tous les types biologiques sont présents. Les Nanophanérophytes (np) et les Microphanérophytes (mp) sont les plus dominants avec une proportion de 32 p.c. chacun tandis les Mégaphanérophytes (MP), les géophytes (G) et les Hémicryptophytes $(H)$ sont très faiblement représentés (Figure 4A). Quant aux Thérophytes (Th), les
Chaméphytes (Ch) et les Mésophanérophytes $(\mathrm{mP})$, ils représentent respectivement 13 p.c., 9 p.c. et 7 p.c. Concernant les types morphologiques, les herbes et les arbustes sont les plus représentés avec respectivement chacun 34 p.c. et 31 p.c. (Figure 4B). Les lianes ont une proportion de 23 p.c. et les arbres avec 12 p.c. sont le type morphologique le moins représenté. La répartition des adventices selon leurs affinités chorologiques montre la prédominance des espèces appartenant à la région guinéo-congolaise avec 37 espèces et, celles appartenant à la zone guinéo-congolaise et soudanozambézienne avec 36 espèces. Carica papaya et Celosia argentea sont les deux espèces introduites. Une espèce Sabicea vogelii appartient au bloc forestier Ouest-africain.

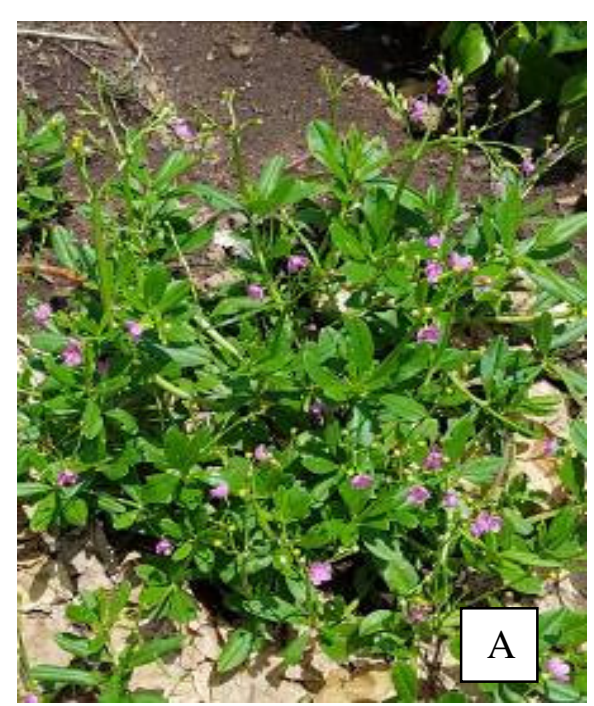

Talinum triangulare (Portulacaceae)

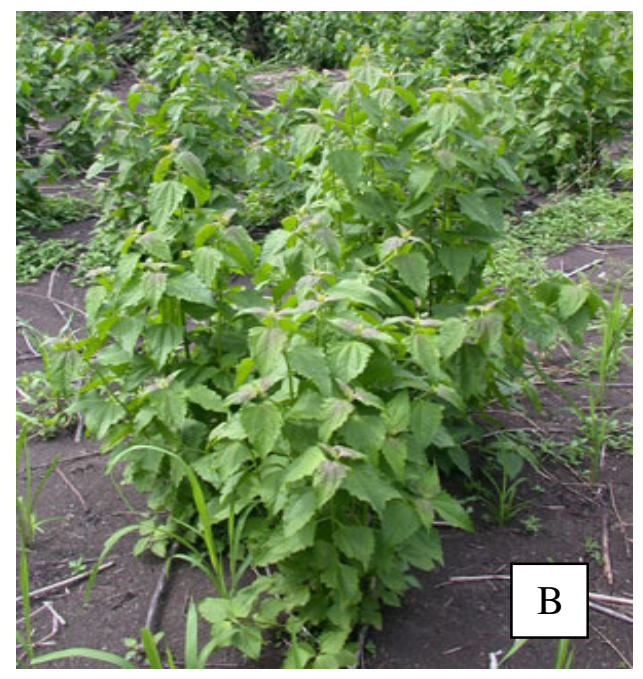

Chromolaena odorata (Asteraceae)

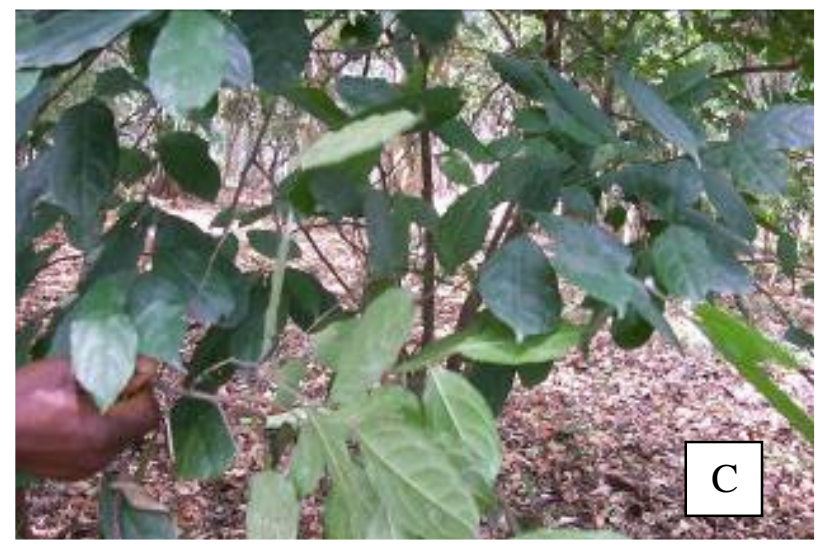

Motandra guineensis (Apocynaceae)

Figure 3 : Illustrations de quelques adventices utilisées dans divers domaines par les populations riveraines de la forêt classée de Sanaimbo 


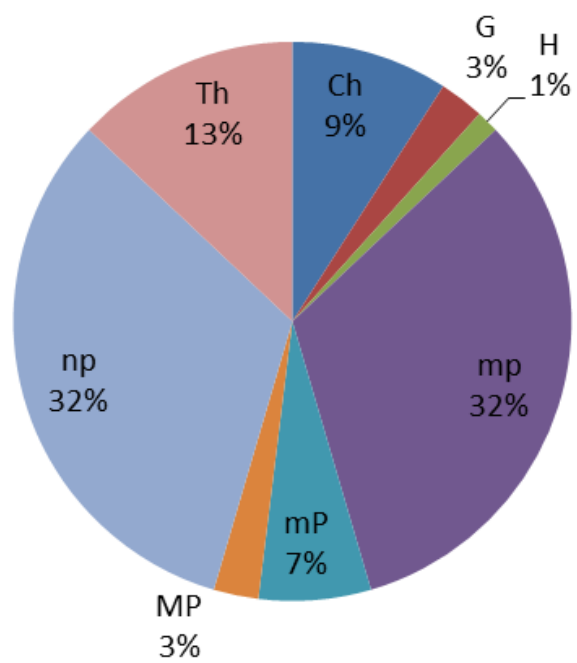

A

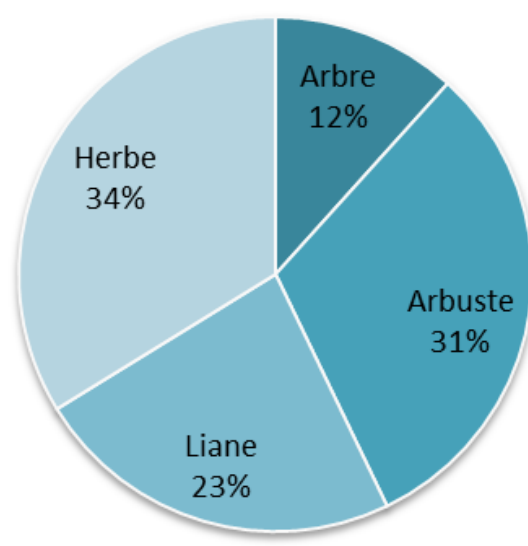

B

Figure 4 : Spectres des types biologiques (A) et des types morphologiques $(B)$ des adventices utilisés par les populations riveraines de la forêt classée de Sanaimbo

\begin{tabular}{|c|c|}
\hline $\begin{array}{l}\text { Différents domaines d'utilisation des plantes } \\
\text { récoltées } \\
\text { Les domaines d'utilisation des adventices sont } \\
\text { divers : l'alimentation, la médecine, la cosmétique, la } \\
\text { chasse, la construction et l'artisanat. Les adventices ont } \\
\text { été classées en } 3 \text { groupes: les adventices utilisées } \\
\text { comme plante alimentaire, celles utilisées en médecine }\end{array}$ & $\begin{array}{l}\text { traditionnelle et celles destinées à d'autres usages. Les } \\
\text { populations des régions d'étude utilisent beaucoup plus } \\
\text { les adventices pour se soigner car la fréquence } \\
\text { d'utilisation des adventices dans la médecine } \\
\text { traditionnelle est de } 79 \text { p.c. tandis qu'elle est de } 11 \text { p.c. } \\
\text { dans l'alimentation et de } 10 \text { p.c. dans les autres usages } \\
\text { (figure 5). }\end{array}$ \\
\hline
\end{tabular}

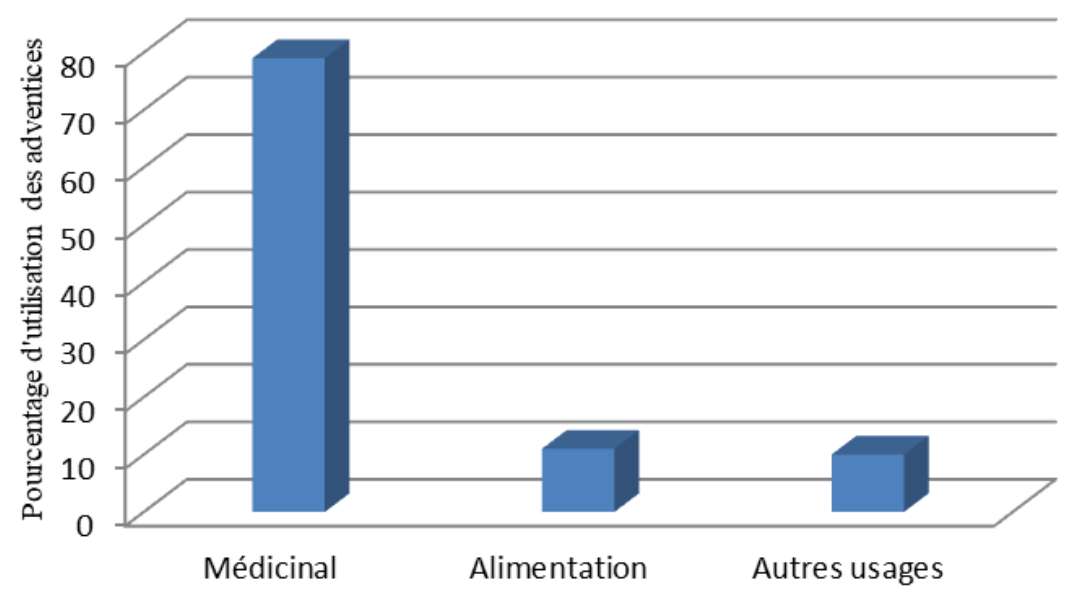

Domaines d'utilisation des adventices

Figure 5 : Fréquence d'utilisation des adventices dans différents domaines par les populations riveraines de la forêt classée de Sanaimbo 

riveraines de la forêt classée de Sanaimbo (centre-est de la Côte d'Ivoire).

Adventices utilisées dans l'alimentation: Les adventices comestibles rencontrées sont au nombre de 10 (tableau 1). Les organes généralement consommés sont les feuilles, les fruits, les tubercules et les tiges. Les fruits de Deinbollia pinnata et de Microdesmis keayana sont consommés crus. Les tubercules de Dioscorea odoratissima cuits à l'eau sont consommés pilés ou bouillis. Les tiges feuillées de Talinum triangulare (figure 3A) sont consommées en sauce. Pour les 6 autres espèces et pour Microdesmis keayana, ce sont les feuilles qui sont consommées en sauce.

Tableau 1 : Adventices consommées par des communautés riveraines de la forêt classée de Sanaimbo.

\begin{tabular}{l|l|l}
\hline Espèces & Nom local & Partie utilisée \\
\hline Cassia obstusifolia & tiguènanflabrou (malinké) & feuilles \\
Celosia argentea & porlonbrou (malinké) & feuilles \\
Deinbollia pinnata & adjépklorwa bacaha (agni) & fruits \\
Dioscorea odoratissima & coca assêh (agni) & tubercules \\
Laportea aestuans & awaflabrou (malinké) & feuilles \\
Microdesmis keayana & Zanzannimo (agni) & feuilles \\
Solanum nigrum & fruits \\
Talinum triangulare & foué n'gna (agni) & feuilles \\
Triplochiton scleroxylon & n'kindapkassin (agni) & tige feuillée \\
Triumfetta rhomboidea & kpatabor (agni) & feuilles \\
\hline
\end{tabular}

Adventices utilisées en médecine traditionnelle: Cinquante-sept adventices ont été reconnues par les populations comme étant des plantes à vertus thérapeutiques. Ainsi, 45 affections sont traitées à partir de 92 recettes à base de ces espèces. Ces plantes médicinales permettent de traiter une ou plusieurs pathologies. Selon le nombre de maladies traitées, 4 groupes d'espèces se différencient. Le premier qui compte 36 espèces regroupe les plantes utilisées pour soigner une seule pathologie. Quatorze espèces constituent le second groupe; ce sont les plantes qui entrent dans les recettes pour guérir 2 maladies. Les 6 espèces du groupe 3 traitent chacune 3 maladies différentes. Le quatrième groupe compte 2 espèces, Althernantera sessilis et de Euphorbia hirta. Althernantera sessilis entre dans la préparation de recettes traitant 4 affections qui sont l'accouchement difficile, le manque de vigueur chez l'enfant, l'anémie et l'hémorragie post couche. C'est la plante entière qui est utilisée pour la préparation de ces différentes recettes. Les racines, les feuilles ou même la plante entière de Euphorbia hirta entrent dans la confection de 4 recettes différentes qui permettent de soigner les règles douloureuses, la diarrhée, la constipation et la gonococcie. II existe des adventices qui entrent dans la composition de différents remèdes pour le traitement d'une seule affection. Le paludisme est la pathologie la plus traitée sur les 45 affections. Neuf recettes entrent dans son traitement. Ces recettes sont issues de 7 espèces qui sont: Gouania longipetala, Hoslundia opposita, Lecaniodiscus cupanioides, Morinda lucida, Oxyanthus racemosus, Phyllanthus amarus et Sabicea vogelii. Les parties utilisées pour la préparation des recettes sont soit l'organisme entier ou les organes tels les tiges, les racines, les écorces et les feuilles qui sont les plus sollicitées. Les affections masculines traitées à partir des adventices sont l'hernie et plus fréquemment l'impuissance sexuelle. Les adventices qui interviennent dans la composition des recettes de ces affections sont Momordica charantia, Sabicea vogelii, Blighia unijugata et Turraea heterophylla. Les adventices qui entrent dans la préparation des remèdes des maladies féminines sont au nombre de 13 et servent à composer 18 recettes. Parmi ces adventices, 5 permettent de soigner chacune 2 affections. Ce sont Aerva lanata, Althernantera sessilis, Boerhavia diffusa, Elytraria marginata et Pothomorphe umbellatum. L'affection féminine, la plus diversement traitée est l'accouchement difficile avec 5 recettes à base de 4 adventices. Les affections infantiles traitées à partir des adventices de la forêt classée de Sanaimbo sont au nombre de 8. Treize espèces entrant dans la composition des recettes. A partir de ces 13 adventices, 14 recettes médicamenteuses sont préparées. L'affection la plus diversement traitée est le manque de vigueur chez l'enfant avec 4 recettes. Althernantera sessilis, Amaranthus spinosus, Amaranthus viridis, Boerhavia diffusa et Cassia occidentalis interviennent dans le traitement de cette affection. Les feuilles de Chromolaena odorata (figure 3B) est également utilisée pour le 

riveraines de la forêt classée de Sanaimbo (centre-est de la Côte d'Ivoire).

traitement des douleurs abdominales et de l'hémorragie externe.

Autres formes d'utilisations des adventices : Douze adventices sont utilisées pour divers usages: l'esthétique, la chasse, l'habitat, l'hygiène bucco-dentaire, les fourrages, la menuiserie, la vannerie et l'emballage des aliments (tableau 2). Les adventices utilisés pour la chasse interviennent dans la pose des pièges. Généralement, ce sont les tiges qui sont utilisées comme ressort des pièges. Les adventices concernés sont Calycobolus africanus et Motandra guineensis (Figure 3C) qui sont des lianes. Dans le domaine de la menuiserie, 3 plantes sont utilisées pour fabriquer des meubles (chaises et tabouret) et du matériel de cuisine (pilon). Les feuilles de Lonchocarpus cyanenscens servent à noircir les gencives et les lèvres. Ceci est un critère de beauté dans nos régions. Pour l'hygiène buccodentaire, les tiges de Carpolobia lutea et Clausena anisata servent à curer les dents. Dans la construction, la tige de Motandra guineensis sert d'attache dans la fixation des bois de charpente. Pour l'alimentation du bétail, les populations de ces localités utilisent fréquemment les feuilles de Millettia zechiana. Les populations se servent des pétioles de Marantochloa cuspidata et Thaumatococcus daniellii et de la tige de Motandra guineensis pour la confection de vans, paniers et nattes. Les feuilles de Marantochloa cuspidata et Thaumatococcus daniellii sont utilisées comme emballage des aliments.

Tableau 2 : Adventices utilisées dans divers domaines par les populations riveraines de la forêt classée de Sanaimbo.

\begin{tabular}{l|l|l|l}
\hline Espèces & Nom local & Domaine d'utilisation & Organe utilisé \\
\hline Calycobolus africanus & erhinkoi & Chasse & Tige \\
Carpolobia lutea & Hygiène dentaire & Tige \\
Clausena anisata & aborvan & Hygiène dentaire & Tige \\
Exobolus patens & yanflèssou & Sevrage des bébés & Feuilles \\
Holarrhena floribunda & sêbê & Menuiserie & Tige \\
Lonchocarpus cyanenscens & galé & Esthétique & Feuilles \\
Marantochloa cuspidata & bêhê & Vannerie & Pétioles \\
Millettia zechiana & & Emballage & Feuilles \\
& & Fourrage & Feuilles \\
Motandra guinensis & Vannerie & Tige \\
Nesogordonia papaverifera & dja gnanman & Habitat & Tige \\
Solanum erianthum & Chasse & Tige \\
Thaumatococcus danielii & koumossi & Menuiserie & Tige \\
& bêhêiserie & Tige \\
\hline
\end{tabular}

Taux d'utilisations des organes végétaux : En général, ce sont les feuilles qui sont les plus utilisées (figure 6) avec un taux de 50p.c. Viennent ensuite les tiges et les racines avec respectivement une proportion de 17p.c. et 13p.c. Les autres parties des adventices tels que les écorces, les fleurs, les fruits, les pétioles, les vrilles et les tubercules sont très faiblement sollicités (figure 6 ) avec des taux qui oscillent entre 4p.c. et 1p.c. Pour certaines adventices, la plante entière est sollicitée: ce sont Althernanthera sessilis, Elytraria marginata, Euphorbia hirta, Gouania longipetala, Momordica charantia, Phyllanthus amarus, Portulaca quadrifida et Sida cordifolia. Pour leur alimentation et leur santé, les populations de cette région utilisent plus les feuilles des adventices. Pour les autres usages, ce sont les tiges qui sont les plus sollicitées. 


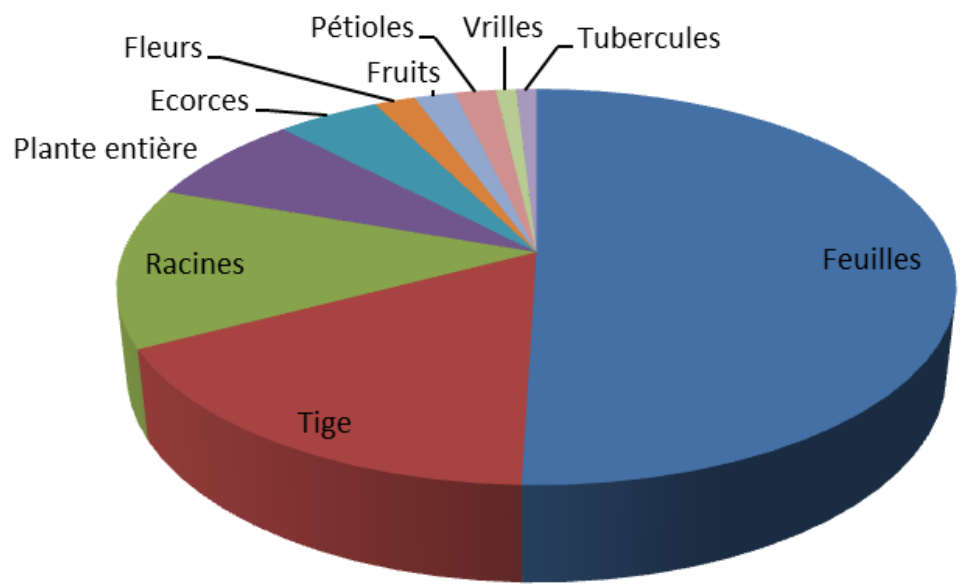

Figure 6 : Spectre de répartition des organes des adventices utilisés dans différents domaines chez les communautés riveraines de la forêt classée de Sanaimbo

\section{DISCUSSION}

Parmi les adventices comestibles, certaines sont consommées dans plusieurs régions de la Côte d'Ivoire. Les études de N'guessan (1995) dans le département d'Agboville ont montré que le peuple krobou consomme également les feuilles de Talinum triangulare et de Triplochiton scleroxylon en sauce. Talinum triangulare est consommée aussi par les populations autochtones du Nord-est de la Côte d'Ivoire (Ouattara et al., 2016). Les jeunes feuilles de Sterculia tragacantha et Triplochiton scleroxylon sont également consommées par les populations du centre-ouest ivoirien (Kouamé et Gnahoua, 2008). Les investigations menées dans le Centre-Ouest et dans l'Ouest de la Côte d'Ivoire (Tra Bi, 1997), ont indiqué que les peuples de ces différentes localités ivoiriennes consomment les fruits de Deinbollia pinnata, les feuilles de Triplochiton scleroxylon et de Solanum nigrum. La valeur nutritive des feuilles de Solanum nigrum a été indiquée par N'dri (1986). Ces feuilles contiendraient 40 p.c. de glucides, 32 p.c. de protéines, 15 p.c. de sels minéraux, 22 oligo-éléments et tous les acides aminés. Selon Aké-Assi (2014), Talinum triangulare et Solanum nigrum font partie des plantes alimentaires de cueillette consommées par les peuples de l'Afrique de l'Ouest. Parmi les adventices utilisées en médecine traditionnelle, certaines sont des rudérales. Les habitants n'ont donc pas toujours besoin d'aller dans leur champ pour en récolter. Ces plantes sont fréquemment rencontrées autour des habitations villageoises, aux abords des sentiers et des rues. Comme espèces rudérales, on cite Ageratum conyzoides, Althernanthera sessilis, Amaranthus viridis, Boerhavia diffusa, Phyllanthus amarus,... Le paludisme est la maladie la plus diversement traitée avec les adventices. Cette maladie constitue un problème majeur de santé publique et la région la plus touchée est l'Afrique subsaharienne (Granado et al., 2006). La Côte d'Ivoire fait partie des quatorze pays d'Afrique où sont recensés quatre-vingt pour cent des décès (Fakih, 2014). Ce qui explique l'importance que les populations de ces localités accordent au traitement de cette pathologie. Boerhavia diffusa, Carica papaya et Cassia occidentalis ont été reconnues comme des plantes utilisées dans la médecine traditionnelle en Afrique de l'Ouest selon Aké-Assi et Guinko (1991). Certaines plantes sont utilisées à des mêmes fins dans des régions différentes. C'est le cas de Boerhavia diffusa qui est utilisée aussi bien par les populations de la zone d'étude (centre-est) que celles du sud (N'guessan et al., 2010) du pays pour faciliter l'accouchement. Cela pourrait s'expliquer par le fait que les peuples autochtones de ces régions appartiennent au même grand groupe ethnique: les Akan. Les autres formes d'utilisation des adventices regroupent la chasse, la menuiserie, l'hygiène bucco-dentaire, la beauté, la vannerie, l'habitat, l'emballage et le fourrage. L'organe le plus utilisé par la population dans ces différents domaines est la tige. Calycobolus africanus et Motandra guineensis sont également utilisées par les peuples du centre-ouest et de l'ouest de la Côte d'Ivoire (Tra Bi, 1997). Leur tige est employée dans la construction (lien) comme c'est le cas du peuple vivant autour de la forêt classée de Sanaimbo. Le mode d'utilisation de la tige de Motandra guineensis est le même pour ces 3 peuples. Dans le centre-ouest du pays plus précisément à Issia, les bété utilisent les feuilles de Motandra guineensis en décoction 
pour traiter le paludisme (Zirihi Guédé et al., 2010). En plus des usages connus que l'on fait de la tige de Calycobolus africanus, les populations de l'ouest l'utilisent comme corde pour sécher le linge. Pour Marantochoa cuspidata et Thaumatococcus daniellii, c'est également les mêmes modes d'usage qu'emploient les populations de ces deux régions ivoiriennes. En plus, dans l'ouest, le centre-ouest (Tra Bi, 1997) et le sud (peuple krobou) (N'guesssan, 1995), leurs feuilles servent de toiture. Le

\section{CONCLUSION}

L'étude des adventices des parcelles cultivées de la forêt classée de Sanaimbo et des agroécosystèmes environnant a permis de mettre en évidence l'utilité de ces espèces considérées nuisibles en agronomie. Les populations riveraines de cette forêt connaissent et utilisent les adventices dans divers domaines. Ces domaines concernent l'alimentation humaine, la médecine traditionnelle et divers autres domaines que sont la chasse, l'esthétique, la vannerie, l'hygiène buccodentaire, le fourrage, la menuiserie et l'emballage. La feuille est l'organe végétal le plus sollicité dans l'alimentation et dans la pharmacopée traditionnelle. Dans les autres domaines, la tige est l'organe végétal le plus sollicité. Les populations de ces quatre localités

\section{REMERCIEMENTS}

Nous tenons à remercier toutes les personnes notamment les agriculteurs des localités visitées, qui nous ont permis d'accéder à leurs champs et ont bien voulu répondre à nos préoccupations. Nous exprimons notre gratitude à la famille

\section{RÉFÉRENCES BIBLIOGRAPHIQUES}

AFNOR: 1977. Produits utilisés en agriculture, agropharmacie : vocabulaire Norme française enregistrée, Association Française de Normalisation. Edition NF U 43-000 ; 1-24.

Aké-Assi L : 2014. Quelques plantes alimentaires de cueillette de l'Afrique de l'Ouest: leur sauvegarde et leur valorisation. Éditions NEICEDA, Abidjan, Côte d'Ivoire. $141 \mathrm{pp}$.

Aké-Assi L. et Guinko S : 1991. Plantes utilisées dans la médecine traditionnelle en Afrique de l'ouest. Editiones Roche Basel, Switzerland, $151 \mathrm{pp}$.

Altieri M. A : 1987. The impact, uses, and ecological role of weeds in agroecosystems. In : Liebman M. and Altieri M. A., 1988. Weed management in agroecosystems : ecological approaches. CRC Press., Inc., Boca Raton, Florida : 1-6.

Fakih Chadi : 2014. Le paludisme en Côte d'Ivoire état des lieux, stratégies de lutte. Thèse pour tronc de Nesogordonia papaverifera sert à la confection des pilons et des mortiers dans toutes ces régions citées. II sert également de charpente chez les populations du centre-ouest et de l'ouest de la Côte d'Ivoire (Tra Bi, 1997). Chromolaena odorata qui est l'adventice la plus invasive des parcelles cultivées de ces localités (Touré et al., 2008), a également des vertus thérapeutiques. Les populations utilisent ses feuilles pour le traitement des douleurs abdominales et de l'hémorragie externe.

(Assahara, Adouakouakro, Soungassi et N'gohinou) emploient beaucoup plus les adventices en médecine traditionnelle qu'en alimentation et dans les autres domaines. Cette étude montre l'importance des plantes adventices dans le quotidien des populations rurales vivant aux alentours de la forêt classée de Sanaimbo et que l'éradication totale de ces plantes en tant qu'adventices ne doit pas être envisagée. C'est pourquoi dans le domaine de la Malherbologie, l'on parle de gestion durable dans la lutte contre les adventices. Car la gestion durable dans la lutte contre ces espèces tient compte de nombreux paramètres que sont l'écologie et la biologie de ces plantes, l'environnement social et économique dans lequel elles se développent.

Aman Kadio de N'gohinou qui nous a hébergés durant notre séjour et également à toutes les personnes qui ont aidé à réaliser ce travail.

l'obtention du Diplôme d'État de Docteur en Pharmacie. Université de Bordeaux (France) U.F.R. des sciences pharmaceutiques $144 \mathrm{pp}$.

Gliessman S. R: 1988. Ecology and management of weeds in traditional agroecosystems. In : Leibman M. and Altieri M., 1988. Weed management in agroecosystems : ecological approaches. CRC Press., Boca Raton, Florida (USA) : 1-6.

Granado S., Ettien A. A.-M., N'Gronma B. N. A., Yao A. K., Tanner M. et Obrist B: 2006. " La vulnérabilité des citadins à Abidjan en relation avec le paludisme ", VertigO - la revue électronique en sciences de l'environnement mis en ligne le 20 décembre 2006, consulté le 28 février 2018: http://journals.openedition.org/ vertigo/1767; DOI : 10.4000/vertigo.1767 
Ipou Ipou J : 2005. Biologie et écologie d'Euphorbia heterophylla L. (Euphorbiaceae) en culture cotonnière, au nord de la Côte d' Ivoire. Thèse de l'Université de Cocody-Abidjan, UFR Biosciences $195 \mathrm{pp}$.

Kouamé N'M-T et Gnahoua GM : 2008. Arbres et lianes spontanés alimentaires du département de Gagnoa (centre-ouest de la Côte d'Ivoire). Bois et Forêts des Tropiques 298 (4) : 65-75.

Kouassi A. M., Okaingni J. C., Kouakou K. E. et Biemi J : 2013. Évaluation des propriétés hydrauliques des aquifères de socle cristallin et cristallophyllien : cas de la région du N'zi-Comoé (Centre-Est de la Côte d'Ivoire). International Journal of Innovation and Applied Studies 2 (1) : 61-71. 〈hal-00781464〉

N'dri P : 1986. Contribution à l'étude de quelques plantes alimentaires spontanées de la région de Divo (Côte d'lvoire). Mémoire de DEA d'Écologie Tropicale, Université d'Abidjan, $65 \mathrm{pp}$.

N'guessan K: 1995. Contribution à l'étude ethnobotanique chez les Krobou de la souspréfecture d'Agboville (Côte d'Ivoire). Thèse 3ème cycle, Univ. Nationale de Côte d'Ivoire, Abidjan 583 pp.

N'guessan K., Zirihi N. G. et Boraud N. K. M : 2010. Étude ethnopharmacologique des plantes utilisées pour faciliter l'accouchement, en pays
Abbey et Krobou, au Sud de la Côte-d'Ivoire. Int. J. Biol. Chem. Sci. 4(4) : 1004-1016.

Ouattara N. D., Gaille E., Stauffer F. W. er Bakayoko A : 2016. Diversité floristique et ethnobotanique des plantes sauvages comestibles dans le Département de Bondoukou (Nord- Est de la Côte d'lvoire). Journal of Applied Biosciences 98 : $9284-9300$.

Ruthenberg H: 1976. Farming systems in the tropics. Clarendon ed. Oxford, $80 \mathrm{pp}$.

Touré A., Ipou Ipou J., Adou Yao C. Y., Boraud M. K. N. et N'guessan K. E : (2008). Diversité floristique et degré d'infestation des mauvaises herbes des agroécosystèmes environnant la forêt classée de Sanaimbo, dans le centre-est de la Côte d'Ivoire. Agronomie Africaine, 20 (1) : 15-25.

Tra Bi F. H : 1997. Utilisation des plantes, par l'homme, dans les forêts classées du Haut-Sassandra et de Scio, en Côte d'Ivoire. Thèse de 3ème cycle, Université de Cocody-Abidjan, Faculté des Sciences et Techniques $215 \mathrm{pp}$.

Zirihi Guédé N., N'guessan K., Etien Dibié T. et Grellier $P: 2010$. Ethnopharmacological study of plants used to treat malaria, in traditional medicine, by Bete Populations of Issia (Côte d'Ivoire). Journal of pharmaceutical Sciences and Research 2 (4) : 216-227. 